\title{
The quadratic hazard model for analyzing longitudinal data on aging, health, and the life span
}

\author{
A.I. Yashin*, K.G. Arbeev, I. Akushevich, A. Kulminski, S.V. Ukraintseva, E. Stallard, \\ K.C. Land
}

Center for Population Health and Aging, Duke University, Durham, NC 27708, United States

Received 16 February 2012; accepted 15 May 2012

Available online 17 May 2012

Communicated by J. Fontanari

\begin{abstract}
A better understanding of processes and mechanisms linking human aging with changes in health status and survival requires methods capable of analyzing new data that take into account knowledge about these processes accumulated in the field. In this paper, we describe an approach to analyses of longitudinal data based on the use of stochastic process models of human aging, health, and longevity which allows for incorporating state of the art advances in aging research into the model structure. In particular, the model incorporates the notions of resistance to stresses, adaptive capacity, and "optimal" (normal) physiological states. To capture the effects of exposure to persistent external disturbances, the notions of allostatic adaptation and allostatic load are introduced. These notions facilitate the description and explanation of deviations of individuals' physiological indices from their normal states, which increase the chances of disease development and death. The model provides a convenient conceptual framework for comprehensive systemic analyses of aging-related changes in humans using longitudinal data and linking these changes with genotyping profiles, morbidity, and mortality risks. The model is used for developing new statistical methods for analyzing longitudinal data on aging, health, and longevity.
\end{abstract}

(c) 2012 Elsevier B.V. All rights reserved.

Keywords: Stochastic process model of aging and mortality; Longitudinal data analysis; Allostatic load; Stress resistance; Physiological norms

\section{Introduction}

Age patterns of human mortality rates demonstrate remarkable regularities in different populations: they decline in childhood, exponentially increase in adult ages, and tend to decelerate and level off at the oldest-old ages. Demographers have developed a number of parametric descriptions of mortality curves capturing all aspects of their variation with age [1-4]. Biodemographers and gerontologists have aimed to explain observed features of mortality curves using emerging theoretical concepts [5-12]. Since the chances of death are affected by internal and external stresses challenging deteriorating defense mechanisms in an aging body, the shape of the age trajectories of mortality curves is likely to reflect the average effects of such deterioration, as well as external influences. In other words, if one

\footnotetext{
* Corresponding author. Tel.: +919668 2713; fax: +9196843861.

E-mail address: aiy@duke.edu (A.I. Yashin).
} 
wants to make conclusions about the aging process developing in individuals comprising the population under study by investigating the age pattern of the corresponding mortality curve, one has to develop a description of such a curve in terms of parameters characterizing the processes which are likely to contribute to the shape of this curve. Such a description can be done using models of aging-related changes represented in longitudinal data merged with data on health and survival events.

Statistical methods for joint modeling of longitudinal and survival (time-to-event) data have been developed during the past several decades using both frequentist and Bayesian approaches (see recent reviews in [13-16] and references therein). Commonly, time-to-event data are analyzed using the proportional hazards model and the usual approach to modeling longitudinal data involves a mixed effects model [17] with the assumption that the random effects or individual-specific parameters are normally distributed, see [18-21] among others). Several papers have developed semiparametric approaches that do not rely on normality of the distribution for the random effects or individual parameters (see, e.g., [22-26]). Further developments include modeling longitudinal data using a stochastic process, either an (integrated) Ornstein-Uhlenbeck or Wiener process, that allows a more flexible description of individual longitudinal dynamics and provides a better fit compared to the usual random effects model (see, e.g., [27-32]).

To be useful as a tool for extracting new information, models of longitudinal data have to be based on realistic assumptions and reflect knowledge and evidence accumulated in the field. Epidemiological studies of risks of disease and death show that the conditional hazards of such events considered as functions of given risk variables often have U- or J-shapes [33-43], so the model of aging-related changes has to take this reality into account. The risk variables as well as their effects on the risks of corresponding events experience aging-related changes (different for distinct individuals) and are measured with certain periodicity in longitudinal studies of aging, health, and longevity. Such data make it possible to evaluate regularities of aging-related changes and their effects on health and survival. An important class of models for joint analyses of longitudinal and time-to-event data incorporating a stochastic process for description of longitudinal measurements is based on this biologically-justified assumption of a quadratic hazard (i.e., U-shaped in general and J-shaped for variables that can take values only on one side of the U-curve) considered as a function of risk factors (i.e., physiological variables). Quadratic hazard models have been developed and intensively applied in studies of longitudinal data [44-48]. The advantage of this approach is that it allows for incorporation of new insights and ideas appearing in the course of research on aging. The prototype of a model discussed in this paper was suggested in [44]. Yashin [45,49] investigated conditions for preserving a Gaussian distribution property of the stochastic covariates under the operation of conditional averaging, and found that model [44] satisfies these conditions. An important property of this model is that the age trajectory of the total mortality rate can be explicitly represented in terms of the first two moments of the conditional distribution of the processes involved in the description of the conditional mortality risk. This property, as well as its flexibility in describing age trajectories of factors affecting conditional risk, make this model a valuable tool for studying aging, health, and longevity using longitudinal data.

Despite the availability of efficient statistical methods for analyzing longitudinal data, and considerable progress in understanding various aspects of human aging, health, and longevity, many facts and research findings remain largely disconnected. Researchers working with data on aging usually deal with small portions of information used for addressing specific research question using standard statistical methods. Such methods, however, largely ignore available knowledge, new research findings, and emerging theoretical concepts about aging in the process of data analyses. As a result, the potential of many large longitudinal datasets, as well as the knowledge accumulated in the field, remains underused.

Progress in this area would be substantially facilitated if researchers had a tool for analyzing the wealth of available data and were able to incorporate important facts, research findings, and emerging theoretical concepts in the analyses. Several such concepts capable of capturing fundamental features of aging-related changes are currently under development. They are related to the notion of allostatic load [50], the decline in adaptive capacity (homeostenosis) [51-54], the decline in resistance to stresses [5,55-57], aging-related physiological norms, and heterogeneity in longitudinal data.

In this paper, we describe a mathematical model for analyzing longitudinal data on aging, health, and mortality that incorporates the four concepts of aging described above (i.e., the notions of age-dependent physiological norms, allostatic load, adaptive capacity, and resistance to stress), review applications of this model to analyses of longitudinal data, and investigate its potential for performing more comprehensive analyses of such longitudinal data.

An initial version of such a model was suggested in [58]. Its various extensions have been applied in different contexts to investigate mechanisms of aging-related changes in connection with morbidity/mortality risks. This includes 
analyses of age trajectories of different physiological indices (such as blood glucose, body mass index, cholesterol, diastolic blood pressure, hematocrit, pulse pressure, and pulse rate) in relation to mortality/morbidity risks [59-62]; applications to "indices of cumulative deficits," which have proved to be useful for analyses of a wide spectrum of information in relation to health- and aging-related changes and better characterize the aging phenotype than chronological age [63]; and analyses of trajectories of medical costs in relation to mortality risks [64]. Extended versions of this model also have been used in analyses of dependent competing risks $[65,66]$, heterogeneity in longitudinal data [67], analyses of genetic effects on age trajectories of physiological indices [68], joint analyses of individual health histories and physiological aging [69], and joint analyses of data collected using different observational plans [70].

\section{Model}

\subsection{General description}

To analyze longitudinal data on age-dependent changes in physiological states, we propose a dynamic model describing the trajectories of the individual physiological variables and their influence on mortality risks, which have the J-, or U-shape considered as a function of risk factors [71]. Let $Y_{t}$ (where $t$ is age) be a $k$-dimensional stochastic process describing a continuously changing vector of risk factors/covariates (e.g., physiological variables), and $Z$ be a vector of time-independent observed covariates (e.g., person's genetic background). We specify the risk function or conditional hazard of death in the form

$$
\mu\left(t, Y_{t}, Z\right)=\mu_{0}(t, Z)+\left(Y_{t}-f_{0}(t, Z)\right)^{T} Q(t, Z)\left(Y_{t}-f_{0}(t, Z)\right) .
$$

Here $\mu_{0}(t, Z)$ is a background hazard characterizing the mortality rate that would remain if the vector of covariates $Y_{t}$ followed the optimal trajectory coinciding with $f_{0}(t, Z)$. " $T$ " in (1) and in formulas below denotes the transposition of the noted vectors or matrices. The matrix $Q(t, Z)$ is a non-negative-definite symmetric matrix of dimension $k \times k$. We use column vectors throughout; thus, the transposition to row vectors in (1) is needed to produce a scalar quadratic term in the hazard rate. The one-dimensional version of (1) is

$$
\mu\left(t, Y_{t}, Z\right)=\mu_{0}(t, Z)+\mu_{1}(t, Z)\left(Y_{t}-f_{0}(t, Z)\right)^{2},
$$

where $\mu_{1}(t, Z)$ is some non-negative function of age, $t$. The $\mathrm{U}$-shape of the risk function may change with increasing age. This is important because the narrowing of the U-shape for some risk factor with age captures the age-related decline in resistance to stresses associated with changes in this factor.

The age trajectory of a physiological variable, for which the minimum value of the risk function is reached, is called the physiological norm. We allow for the norm to be a function of age.

Temporal changes in the vector of risk factors $Y_{t}$ are described by a diffusion type stochastic differential equation:

$$
d Y_{t}=a(t, Z)\left(Y_{t}-f_{1}(t, Z)\right) d t+b(t, Z) d W_{t},
$$

with initial condition $Y_{0}$. Here $W_{t}$ is specified as a vector Wiener process with independent components, which describes exogenous challenges affecting these covariates. The process $W_{t}$ is assumed to be independent of the initial vector $Y_{0}$ and covariate $Z$ with normally distributed components. Note that such a model preserves the Gaussian property: in the case of an initial Gaussian distribution for $Y_{0}$, i.e., in the case that the vector of risk factors observed/measured at the initial wave of a longitudinal study is distributed as a Gaussian random variable, the distribution of $Y_{t}$ among survivors is also Gaussian [45,46,49]. Thus, our assumption of a Gaussian initial distribution defines the structure of the entire process $Y_{t}$. The strength of disturbances of $W_{t}$ is characterized by a matrix of diffusion coefficients $b(t, Z)$.

The vector-function $f_{1}(t, Z)$ (with the same dimension as the vector $Y_{t}$ ) describes a trajectory of physiological states that organisms subject to allostasis [72] are forced to follow by the process of adaptive regulation at age $t$. Allostasis is the process of individual adaptation to persistent external disturbances aimed at achieving stability, or homeostasis, through physiological or behavioral change.

The trajectory of $Y_{t}$ reflects aging-related changes in the organism's functioning due to the average effects of a complicated interplay among the ontogenetic program, senescence, and environmental stresses. Persistent external or internal disturbances affect age trajectories of the physiological indices. This is called the effect of allostatic adaptation. Taking into account this effect is especially important in analyses of longitudinal data in which measurements of 
external disturbances are absent or limited. The effects of allostatic adaptation produce deviations from the norm in the trajectories of the process $Y_{t}$. The magnitudes of such deviations for each physiological index will be associated with components of allostatic load.

Homeostatic regulation plays a fundamental role for living organisms and such regulation needs to be included in the equation describing physiological changes. The dynamic model (3) includes a description of negative feedback mechanisms with coefficients of homeostatic regulation given by a matrix $a(t, Z)$. According to (3), the age trajectory of physiological variables $Y_{t}$ will tend to follow the function $f_{1}(t, Z)$, i.e., adapt to changes in $f_{1}(t, Z)$ (the absence of such negative feedback mechanism would allow the trajectories to deviate from $f_{1}(t, Z)$ indefinitely, which is biologically implausible). The ability to adapt depends on the absolute values of the coefficients that are components of the matrix $a(t, Z)$. Age-related changes in these coefficients characterize changes in adaptive capacity with age. Specifically, the elements of the matrix $a(t, Z)$ regulate the age trajectories of the components of the physiological state approximated by the vector $Y_{t}$, i.e., they characterize the rate of the adaptive response for any deviation of a physiological index from the state $f_{1}(t, Z)$ which an organism tends to follow. An important feature of aging - the decline in adaptive capacity - has never been measured directly in longitudinal studies of aging, health, and longevity before. The use of the matrix $a(t, Z)$ in our model allows us to evaluate this effect. For example, in a simplified one-dimensional case, when $b(t, Z)=0$, for all $t$, in Eq. (3), and constant negative $a(t, Z)=a$ for all $t$, the parameter $a$ is the coefficient of negative feedback in the equation for $Y_{t}$, which keeps the trajectory $Y_{t}$ close to $f_{1}(t, Z)$. When $f_{1}(t, Z)=f_{1}$, constant for all $t$ and $Z$, the value of $Y_{t}$ asymptotically approaches $f_{1}$. In the case of non-zero disturbances, the higher the absolute value of $a$, the closer $Y_{t}$ is to $f_{1}$, and the faster $Y_{t}$ tends to $f_{1}$. That is why the value $a(t, Z)$ characterizes adaptive capacity. When the absolute value of the coefficient $a(t, Z)$ declines with age, more time is needed for the trajectory of $Y_{t}$ to approach $f_{1}(t, Z)$ at old ages compared to younger ages. Practical estimation of the changes in adaptive capacity with age involves maximization of the likelihood function (6) below, in which coefficients of matrix $a(t, Z)$ are described as parametric functions of age.

The vector-function $f_{0}(t, Z)$ in (1) (or, correspondingly, the scalar function $f_{0}(t, Z)$ in (2)) is introduced to explicitly characterize age-related changes in the "optimal" physiological state corresponding to the minimum hazard at a given age. It represents the age-dependent norm for a given functional state. It may differ from $f_{1}(t, Z)$ since the process of allostatic adaptation does not necessarily result in the optimal physiological state. Thus, the difference between $f_{1}(t, Z)$ and $f_{0}(t, Z)$ provides a measure of the allostatic load.

\subsection{Estimation procedure}

The model can be estimated using the maximum likelihood method. The survival function associated with the life span distribution is $P(X>t \mid Z)=\exp \left(-\int_{0}^{t} \bar{\mu}(u, Z) d u\right)$, where the marginal (unconditional) hazard $\bar{\mu}(u)$ has the form (see $[47,65,73])$ :

$$
\bar{\mu}(u, Z)=\mu_{0}(u, Z)+\left(m(u, Z)-f_{0}(u, Z)\right)^{T} Q(u, Z)\left(m(u, Z)-f_{0}(u, Z)\right)+\operatorname{Tr}(Q(u, Z) \gamma(u, Z))
$$

where $\operatorname{Tr}(\cdot)$ denotes the matrix trace operator and $m(u, Z)$ and $\gamma(u, Z)$ satisfy the following system of ordinary non-linear differential equations:

$$
\begin{aligned}
& \frac{d m(t, Z)}{d t}=a(t, Z)\left(m(t, Z)-f_{1}(t, Z)\right)-2 \gamma(t, Z) Q(t, Z)\left(m(t, Z)-f_{0}(t, Z)\right), \\
& \frac{d \gamma(t, Z)}{d t}=a(t, Z) \gamma(t, Z)+\gamma(t, Z) a(t, Z)^{T}+b(t, Z) b(t, Z)^{T}-2 \gamma(t, Z) Q(t, Z) \gamma(t, Z),
\end{aligned}
$$

with $m(0, Z)$ and $\gamma(0, Z)$ being the mean and the variance/covariance matrix of the conditional normal distribution of the initial vector $Y_{0}$, given $Z$. Note that in such a model the conditional distribution of $Y_{t}$ among survivors is also Gaussian at any age $t[45,46,49]$. The mean and the variance/covariance matrix of this distribution at age $t$ are given by $m(t, Z)$ and $\gamma(t, Z)$, respectively.

Let the sequence $y_{t_{0}^{i}}^{i}, y_{t_{1}^{i}}^{i}, \ldots, y_{t_{i}^{i}}^{i}, \tau_{i}$ represent the results of measurements of the process $Y_{t}$ and the life span (which may be censored) related to the $i$ th individual. The conditional likelihood function for $N$ individuals is (see $[47,65,73])$ : 


$$
\begin{aligned}
& \prod_{i=1}^{N} \bar{\mu}^{i}\left(\tau_{i}, Z\right)^{\delta_{i}} \exp \left\{-\int_{0}^{\tau_{i}} \bar{\mu}^{i}(u, Z) d u\right\} \prod_{j=0}^{n_{i}\left(\tau_{i}\right)}(2 \pi)^{-\frac{k}{2}}\left|\gamma^{i}\left(t_{j}^{i}-, Z\right)\right|^{-\frac{1}{2}} \\
& \quad \times \exp \left\{-\frac{1}{2}\left(y_{t_{j}^{i}}^{i}-m^{i}\left(t_{j}^{i}-, Z\right)\right)^{T} \gamma^{i}\left(t_{j}^{i}-, Z\right)^{-1}\left(y_{t_{j}^{i}}^{i}-m^{i}\left(t_{j}^{i}-, Z\right)\right)\right\} .
\end{aligned}
$$

Here the superscript " $i$ " denotes respective characteristics for $i$ th individual, $\delta_{i}$ is a censoring indicator, $m^{i}(t, Z)$ and $\gamma^{i}(t, Z)$ satisfy Eqs. (5) at the intervals $\left[t_{0}^{i}, t_{1}^{i}\right) ;\left[t_{1}^{i}, t_{2}^{i}\right) ; \ldots ;\left[t_{n_{i}-1}^{i}, t_{n_{i}}^{i}\right) ;\left[t_{n_{i}}^{i}, \tau_{i}\right)$ with the initial conditions $y_{t_{0}^{i}}^{i}, y_{t_{1}^{i}}^{i}, \ldots, y_{t_{n_{i}}^{i}}^{i}$, respectively. Here $m^{i}\left(t_{j}^{i}-, Z\right)=\lim _{t \uparrow t_{j}^{i}} m^{i}(t, Z)$, and $\gamma^{i}\left(t_{j}^{i}-, Z\right)=\lim _{t \uparrow t_{j}^{i}} \gamma^{i}(t, Z)$, and $t_{n_{i}}^{i}$ is the age of the latest measurement of a functional state before death/censoring at $\tau_{i}$.

Maximization of this likelihood function is computationally extensive, because it involves solution of the systems of ordinary differential equations (ODE) (5) for each measurement, for each individual, and at each step of the likelihood optimization procedure. However, our experience with these models is that such solutions are feasible on modern computers and using modern statistical and technical software, e.g., MATLAB's Optimization Toolbox and ODE solvers, or SAS PROC NLP, implementing different optimization algorithms (such as the Newton-Raphson or trustregion methods) and the Runge-Kutta method for the ODE solution. The parameter estimates then characterize the dynamics of the stochastic process $Y_{t}$ describing the trajectories of physiological aging, as well as changes in mortality risk over age.

Note that the observed values $y_{t_{0}^{i}}^{i}, y_{t_{1}^{i}}^{i}, \ldots, y_{t_{n_{i}}^{i}}^{i}$ are used as initial conditions for differential equations (5) at the beginning of subsequent intervals between the observation times. Therefore, the individual trajectories of $m^{i}(t, Z)$ and $\gamma^{i}(t, Z)$ differ even for individuals having the same values of $Z$. Consequently the estimates of the chances of death for individuals having different observed values of the respective covariates also differ.

In this version of the model, we assume that observations $y_{t_{0}^{i}}^{i}, y_{t_{1}^{i}}^{i}, \ldots, y_{t_{n_{i}}^{i}}^{i}$ do not contain measurement errors. This assumption is not a serious problem when the model's parameters are used to characterize the entire population of study participants. In this case one random process describes age trajectories of functional state in the population. The individual trajectories are just sampling paths of this process, so the differences among individuals are generated by the Wiener process and difference in values at the age at entry into the study. This rough approximation is appropriate for evaluating and predicting population characteristics (e.g., changes in distributions of aging, health, and survival indices in the population in response to changes in health care policy, modification in Medicare services, etc.). It, however, may be unsatisfactory in applications dealing with "personalized" analyses when one is more concerned about the response of individual characteristics to preventive measures or medical interventions. In this situation, one has to "track" individual age trajectories of physiological states, i.e., use different model parameters to describe the age trajectories of the functional states for different individuals. In such individualized applications, taking the measurement error into account could be an important issue. The model can easily be extended to this case. The random process described by Eq. (3) can be personalized, i.e., (ideally) each individual can be characterized by his/her own stochastic process independent of the processes describing the other individuals.

Since the conditional distribution of $Y_{t}$ among survivors is Gaussian, whereas most of physiological indices that can be represented by $Y_{t}$ are positive, and because the model's characteristics have to be biologically interpreted, appropriate constraints on the parameters of the model are necessary in the estimation procedure. In particular, a) the distribution of $Y_{0}$ should guarantee a negligible probability of negative values; b) the functions $f_{1}(t, Z)$ and $f_{0}(t, Z)$ should have non-negative values for each age; c) the absolute values of feedback coefficients in the matrix $a(t, Z)$ in (3) should not become too small for the trajectories of $Y_{t}$ to tend to $f_{1}(t, Z)$; d) the background hazard $\mu_{0}(t, Z)$ should have non-negative values for each age and a non-decreasing age pattern; and e) the matrix $Q(t, Z)$ should remain non-negative definite for each age. Our practical experience with these models shows that such constraints usually do not impose limitations in applications of models to real data. An alternative approach would involve the use of the geometric Brownian motion (GBM) for description of trajectories of physiological variables. GBM is, for example, the most popular model used in financial mathematics for description of stock price dynamics (see, e.g., the works by Samuelson [74], Black and Scholes [75], and Merton [76], and numerous developments and applications of this theory in the later decades). GBM ensures that the modeled trajectories are non-negative, which is the desired property for trajectories of physiological variables. Such considerations, however, are beyond the scope of this paper. 


\subsection{Simulation studies}

We generated 100 datasets with data on life spans and a hypothetical physiological index (mimicking pulse pressure) for 5000 individuals in each dataset. We followed individuals for 56 years with 28 biennial observations of physiological indices, with ages at entry to the study uniformly distributed over the age interval [30,60]. Individuals with life spans exceeding the age at entry plus duration of the follow-up period (56 years) were considered censored at this age. Such a data structure is similar to the Framingham Heart Study original cohort data [77,78]. We estimated the one-dimensional version of the model (Eqs. (2), (3)) with a constant diffusion coefficient (function $b$ ), $\sigma_{1}$, linear functions of age $(t)$ for the quadratic hazard term (function $\mu_{1}$ ), the adaptive capacity (i.e., the negative feedback coefficient $a$ ), and the physiological "norm" (function $f_{0}$ ): $\mu_{1}(t)=a_{\mu_{1}}+b_{\mu_{1}} t, a(t)=a_{Y}+b_{Y}(t-30)$, $f_{0}(t)=a_{f_{0}}+b_{f_{0}}(t-30)$, and a Gompertz function for the baseline hazard: $\ln \mu_{0}(t)=\ln a_{\mu_{0}}+b_{\mu_{0}}(t-30)$ (note that for simplicity all these characteristics are assumed independent of covariates $(Z)$ ). The "allostatic trajectory" (function $f_{1}$ ) was assumed dependent on age $(t)$ and a dichotomous covariate $Z(Z=(0,1) ; P(Z=1)=0.5)$ : $f_{1}(t, Z)=a_{f_{1}}+b_{f_{1}}(t-30)+\beta_{f_{1}} Z$. The initial value of the diffusion process for $Y_{t}, Y_{0}$, was assumed normal, $Y_{0} \sim N\left(f_{1}\left(t_{0}, Z\right), \sigma_{0}\right)$, where $t_{0}$ is the age at the baseline exam for the respective individual. Initial values of the parameters are given in Table 1 in the row labeled True Values.

The results of simulations are summarized in Table 1 and Fig. 1. The simulations confirm that the parameters of the models can be estimated with reasonable accuracy for a sample size of this magnitude.

\section{Discussion}

In experimental studies of aging using populations of laboratory animals, the sensitivity of the individual aging process to external disturbances (e.g., medical interventions), or genetic manipulations often is evaluated by comparing empirical survival functions (or mortality rates) constructed for populations in the experimental and control groups. Similarly, the slope of the logarithm of the mortality curve at the adult and old ages often is interpreted as the aging rate [79]. The limitations of such an interpretation have been discussed in a number of papers. Yashin et al. [80] argued that the use of such a measure of the aging rate may be misleading: the changes in the slope of the mortality curve may occur for many other reasons having little to do with the aging process. For example, the slope could change as a result of changes in the heterogeneity distribution (e.g., distribution of frailty $[81,82]$ ), saving individuals' lives by providing necessary medical help in cases of emergency, etc. Analyzing changes in mortality rates in developed countries, Rozing and Westendorp [83] came to the conclusion that recent progress in mortality reduction does not affect the slope of the logarithm of the mortality curves (see also [80] and references therein for similar observations). Koopman et al. [84] criticized the use of the slope of the logarithm of the mortality curve as a measure of the aging rate and proposed, instead, the use of the age-derivative of the mortality rate as such a measure. Although the use of the derivative overcomes some limitations of the slope measure, it could also be criticized because it does not link changes in mortality with biological changes in the aging human body. Data on many such changes affecting health and survival events are collected in longitudinal data. That is why in situations characterized by the wide spectrum of longitudinal data and findings on aging, focusing solely on the properties of the mortality curve and ignoring the presence of other relevant information about aging-related changes affecting survival chances is scientifically incomplete.

The need for the development of new models of aging, health, and mortality and for describing their connection with traditional demographic models has been emphasized by Manton and Yashin [48]. The connection between the Gompertz model of the mortality curve with the model describing longitudinal data played an important role in better understanding forces and mechanisms shaping the age pattern of the demographic mortality rate (see [48] and references therein). This connection is described by the conditional mortality rate in the form of a generalized Gompertz model:

$$
\mu\left(t, Y_{t}\right)=\tilde{Y}_{t}^{T} \tilde{Q} \tilde{Y}_{t} e^{\theta t}
$$

where $\tilde{Y}_{t}^{T}=\left(1, Y_{t}^{T}\right)$ is a vector of covariates $Y_{t}$ (e.g., physiological indices), $t$ is age, $\tilde{Q}$ is a (constant) matrix, and $\theta$ is the Gompertz exponential growth parameter [48]. In applications of this model to longitudinal data, the estimated value of the parameter $\theta$ has always been smaller than the corresponding parameter in a Gompertz model that does not include information on covariates. The reduction of the exponential growth parameter has been interpreted as 
Table 1

Means, standard deviations (St. dev.) and minimal and maximal values of parameter estimates in 100 simulated data sets.

\begin{tabular}{|c|c|c|c|c|c|c|c|c|c|c|c|c|c|}
\hline & $\ln a_{\mu_{0}}$ & $b_{\mu_{0}}$ & $a_{\mu_{1}} \cdot 10^{4}$ & $b_{\mu_{1}} \cdot 10^{5}$ & $a_{Y}$ & $b_{Y} \cdot 10^{3}$ & $\sigma_{0}$ & $\sigma_{1}$ & $a_{f_{1}}$ & $b_{f_{1}}$ & $a_{f_{0}}$ & $b_{f_{0}}$ & $\beta_{f_{1}}$ \\
\hline Mean & -7.009 & 0.080 & 1.486 & 0.305 & -0.200 & 0.990 & 6.008 & 5.001 & 54.986 & 0.201 & 50.016 & 0.200 & 4.992 \\
\hline St. dev. & 0.144 & 0.003 & 0.364 & 0.062 & 0.004 & 0.142 & 0.060 & 0.016 & 0.136 & 0.005 & 0.393 & 0.013 & 0.137 \\
\hline Min & -7.321 & 0.074 & 0.583 & 0.169 & -0.212 & 0.671 & 5.856 & 4.961 & 54.617 & 0.189 & 49.044 & 0.156 & 4.673 \\
\hline Max & -6.705 & 0.087 & 2.256 & 0.468 & -0.188 & 1.432 & 6.168 & 5.042 & 55.287 & 0.211 & 51.156 & 0.232 & 5.441 \\
\hline True Values & -7.0 & 0.08 & 1.5 & 0.3 & -0.2 & 1.0 & 6.0 & 5.0 & 55.0 & 0.2 & 50.0 & 0.2 & 5.0 \\
\hline
\end{tabular}



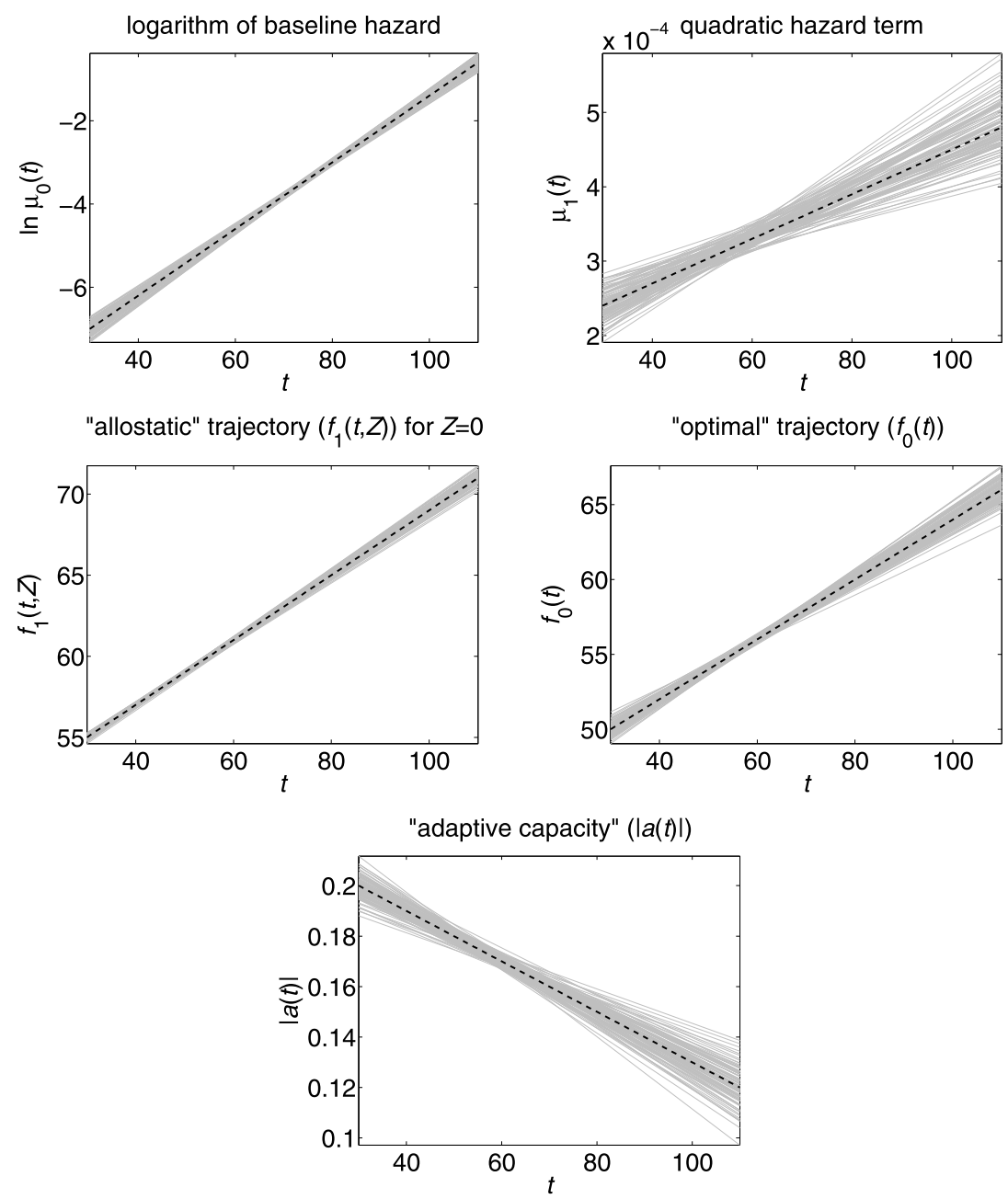

Fig. 1. Estimated (solid grey lines) and true (dashed black lines) trajectories in 100 simulated data sets.

an effect of measurements: the new (reduced) value of the parameter $\theta$ characterized the unexplained component of aging-related increase in the mortality rate. Versions of this model have been applied to several datasets.

For example, Manton et al. [71] applied the generalized Gompertz model in (7) to longitudinal data from the first 18 biennial examinations of the Framingham Heart Study (FHS) and compared the results with those obtained from the first 3 waves of the National Long Term Care Survey (NLTCS: 1982, 1984, and 1989). The use of 10 cardiovascular covariates in the FHS model reduced the $\theta$-parameters from $9.4 \%$ to $8.1 \%$ for males and from $10.0 \%$ to $8.1 \%$ for females. The FHS study population ranged from 30 to 60 years at the start of the 34-year observation period; hence, the reduced $\theta$-parameters implied that the FHS mortality rates would double every 8.6 years if one were to hold the observed covariates constant at their age-30 values.

The use of 27 ADL, IADL, and physical performance covariates in the NLTCS model reduced the $\theta$-parameters from $8.2 \%$ to $5.3 \%$ for males and from $9.1 \%$ to $4.8 \%$ for females. The NLTCS study population was 65 years or older at each wave; hence, the reduced $\theta$-parameters implied that the mortality rates would double every 13.1 or 14.6 years (males, females) if one were to hold the observed covariates constant at their age-65 values. The relative reductions in $\theta$ and the corresponding increases in the doubling times were larger for the NLTCS model, indicating that the NLTCS covariates explained more of the age-dependence of mortality than the FHS covariates explained. The differences were attributable, in part, to the age patterns of several FHS covariates which reached peak values near age 60, followed by declines at older ages [61]. 
The conditional hazard rate (7) in the one-dimensional version of the original model can be represented as follows:

$$
\mu\left(t, Y_{t}\right)=\left(\mu_{0}+\mu_{1}\left(Y_{t}-c\right)^{2}\right) e^{\theta t}=\mu_{0} e^{\theta t}+\mu_{1} e^{\theta t}\left(Y_{t}-c\right)^{2},
$$

where $\mu_{0}, \mu_{1}$, and $c$ are constants. The term $\left(\mu_{0}+\mu_{1}\left(Y_{t}-c\right)^{2}\right)$ in (8) describes one of the parameters of the Gompertz mortality model explained by observations $Y_{t}$. Thus, Eq. (8) can be interpreted as providing a more detailed description of the Gompertz mortality curve widely used in demography. In another interpretation, the term $\mu_{0} e^{\theta t}$ is interpreted as the "optimal" Gompertz mortality rate which would be observed in an ideal situation when $Y_{t} \equiv c$. This representation clearly shows the limitations of the original version: (i) the exponential multipliers in both components of the risk function are the same; (ii) the minimum of the second (quadratic hazard) term is reached at a constant level of the observed covariates.

In the generalized hazard model (1) described above, the covariates' values minimizing the risk function can change over age. This is a more realistic assumption since in epidemiologic and medical practice specialists often use the notion of physiological "norm", specific to a given age. This age-dependent norm is explicitly included in the description of age trajectories of mortality risk (compare Eqs. (1), (2), and (8)). This allows one to statistically test hypotheses about age-dependence of physiological norms and verify such dependence from available data. The modified hazard model (1) includes the earlier version (8) as a particular case.

In the generalized model, the term $\mu_{0}(t)$ can differ from the multiplier of the quadratic hazard $\mu_{1}(t)$ (we omit dependence of these functions on $Z$ to make this case comparable with (8)) which results in a completely new interpretation of these coefficients. The hazard rate $\mu_{0}(t)$ is associated with death from unmeasured factors. The risk $\mu_{0}(t)$ must be smaller than the total (demographic) mortality risk $\bar{\mu}(t)$ calculated in the absence of observations on risk factors. Therefore, $\mu_{0}(t)$ characterizes the mortality remaining after all observed covariates follow the "optimal" trajectory and its interpretation remains similar to that used in the original model. In case of a Gompertz specification, both Gompertz parameters in $\mu_{0}(t)$ can be evaluated and compared with their values in $\bar{\mu}(t)$. This model allows for evaluating the gain in life expectancy when observed covariates follow normal age trajectories.

The term $\mu_{1}(t)$ clarifies the connections between senescence, longevity, and stress-resistance. Indeed, the increasing pattern of $\mu_{1}(t)$ indicates that the branches of the respective U-shaped risk function get steeper, and the range of tolerant deviations of the resultant risk factor from its "optimal" value becomes narrower with age, reflecting the decline in stress resistance with age. Although many aspects of such connections have been investigated in experimental animal studies [56], they have never been adequately addressed in studies of human longitudinal data. Since the decline in stress resistance is an important indicator of aging (senescence), the rate of increase in $\mu_{1}(t)$ (not the slope of the logarithm of the mortality curve) may characterize the rate of aging. More generally, the increasing role of senescence in mortality risk could be captured by the widening pattern of the U-shape of the relative risk, which would indicate a faster increase in $\mu_{0}(t)$ compared to $\mu_{1}(t)$. An important methodological advance of the extended model is that it is transformed to a form where effects of senescence on survival, longevity, and disease development may be evaluated from longitudinal data.

Taking into account the age-dependence of the functions $f_{1}(t, Z), f_{0}(t, Z)$, and $a(t, Z)$ allows for testing hypotheses about factors and mechanisms affecting the dynamic properties of the age trajectories of physiological states. For example, one could test whether $f_{1}(t, Z)$ coincides with $f_{0}(t, Z)$. The difference in these functions means that the processes of allostatic adaptation in response to persistent external disturbances do not tend to minimize mortality risk. One can also test the hypothesis about declines in "adaptive capacity" in the aging human body and evaluate patterns of such declines. All such hypotheses can be tested using the likelihood ratio test. For example, to test the hypothesis about the decline in adaptive capacity with age, one needs to estimate the model first with a decline (say, a linear decline with age in $a(t, Z)$ ) and then the model without such a decline (i.e., $a(t, Z)$ is independent of age), where all other functions (except $a(t, Z)$ ) are specified similarly in both models, and compare the likelihood functions in these two models using the likelihood ratio test.

The use of observed fixed covariates $Z$ in the functions $f_{0}(t, Z), Q(t, Z), a(t, Z), b(t, Z)$, and $f_{1}(t, Z)$ makes the model more personalized. The notion of the "norm" may differ for individuals carrying different alleles or genotypes, or having different histories of events and processes experienced by an individual during the life course (e.g., diseases, environmental exposures), etc. These indicate the need for developing a more general methodology, which could incorporate individualized notions of "norms" and adaptive responses.

An important feature of the model discussed above is the preservation of the Gaussian property in the operation of conditional averaging. If the distribution of risk factors at the initial wave of observations, $Y_{0}$, is Gaussian, the 
distribution of $Y_{t}$ among survivors is also Gaussian. This allows for describing the probability distributions of dynamic covariates in terms of the first two moments which satisfy ordinary non-linear differential equations. Note that the Gaussian distribution allows for negative values of the risk factors to occur with positive probability. Our studies show that in practice this property does not limit the analyses. The model can also be used as two-moment approximation for the age trajectories of covariates which follow non-Gaussian dynamics. In non-Gaussian cases, the model could also be extended to include higher order moments (e.g., conditional semi-invariants).

\section{Acknowledgements}

The work was partially supported by grants R01AG030612, R01AG030198 and R01AG032319 from the National Institute on Aging. The content is solely the responsibility of the authors and does not necessarily represent the official views of the National Institute on Aging or the National Institutes of Health.

\section{References}

[1] Heligman L, Pollard JH. The age pattern of mortality. J Inst Actuar 1980;107:49-80.

[2] Mode CJ, Busby RC. An 8-parameter model of human mortality - the single decrement case. Bull Math Biol 1982;44:647-59.

[3] Siler W. Parameters of mortality in human populations with widely varying life spans. Stat Med 1983;2:373-80.

[4] Gage TB. Causes of death and the components of mortality: testing the biological interpretations of a competing hazards model. Amer J Hum Biol 1991;3:289-300.

[5] Strehler BL, Mildvan AS. General theory of mortality and aging. Science 1960;132:14-21.

[6] Charlesworth B. Patterns of age-specific means and genetic variances of mortality rates predicted by the mutation-accumulation theory of ageing. J Theoret Biol 2001;210:47-65.

[7] Gavrilov LA, Gavrilova NS. The reliability theory of aging and longevity. J Theoret Biol 2001;213:527-45.

[8] Lee RD. Rethinking the evolutionary theory of aging: transfers, not births, shape social species. Proc Natl Acad Sci USA 2003;100:9637-42.

[9] Zheng H, Yang Y, Land KC. Heterogeneity in the Strehler-Mildvan general theory of mortality and aging. Demography 2011;48:267-90.

[10] Yashin AI, Iachine IA, Begun AS. Mortality modeling: a review. Math Popul Stud 2000;8:305-32.

[11] Yashin AI, Begun AS, Boiko SI, Ukraintseva SV, Oeppen J. The new trends in survival improvement require a revision of traditional gerontological concepts. Exp Gerontol 2001;37:157-67.

[12] Yashin AI, Begun AS, Boiko SI, Ukraintseva SV, Oeppen J. New age patterns of survival improvement in Sweden: do they characterize changes in individual aging? Mech Ageing Dev 2002;123:637-47.

[13] Tsiatis AA, Davidian M. Joint modeling of longitudinal and time-to-event data: an overview. Statist Sinica 2004;14:809-34.

[14] Yu MG, Law NJ, Taylor JMG, Sandler HM. Joint longitudinal-survival-cure models and their application to prostate cancer. Statist Sinica 2004;14:835-62.

[15] Ibrahim JG, Chu H, Chen LM. Basic concepts and methods for joint models of longitudinal and survival data. J Clin Oncol 2010;28:2796-801.

[16] Sousa I. A review on joint modelling of longitudinal measurements and time-to-event. REVSTAT 2011;9:57-81.

[17] Laird NM, Ware JH. Random-effects models for longitudinal data. Biometrics 1982;38:963-74.

[18] Faucett CL, Thomas DC. Simultaneously modelling censored survival data and repeatedly measured covariates: a Gibbs sampling approach. Stat Med 1996;15:1663-85.

[19] Wulfsohn MS. Tsiatis AA. A joint model for survival and longitudinal data measured with error. Biometrics 1997;53:330-9.

[20] Ibrahim JG, Chen MH, Sinha D. Bayesian methods for joint modeling of longitudinal and survival data with applications to cancer vaccine trials. Statist Sinica 2004;14:863-83.

[21] Xu J, Zeger SL. The evaluation of multiple surrogate endpoints. Biometrics 2001;57:81-7.

[22] Tsiatis AA, Davidian M. A semiparametric estimator for the proportional hazards model with longitudinal covariates measured with error. Biometrika 2001;88:447-58.

[23] Song X, Davidian M, Tsiatis AA. A semiparametric likelihood approach to joint modeling of longitudinal and time-to-event data. Biometrics 2002;58:742-53.

[24] Song XA, Davidian M, Tsiatis AA. An estimator for the proportional hazards model with multiple longitudinal covariates measured with error. Biostatistics 2002;3:511-28.

[25] Brown ER, Ibrahim JG. A Bayesian semiparametric joint hierarchical model for longitudinal and survival data. Biometrics 2003;59:221-8.

[26] Song X, Wang CY. Semiparametric approaches for joint modeling of longitudinal and survival data with time-varying coefficients. Biometrics 2008;64:557-66.

[27] Taylor JMG, Cumberland WG, Sy JP. A stochastic model for analysis of longitudinal AIDS data. J Am Statist Assoc 1994;89:727-36.

[28] Lavalley MP, Degruttola V. Models for empirical Bayes estimators of longitudinal CD4 counts. Stat Med 1996;15:2289-305.

[29] Wang Y, Taylor JMG. Jointly modeling longitudinal and event time data with application to acquired immunodeficiency syndrome. J Am Statist Assoc 2001;96:895-905.

[30] Henderson R, Diggle P, Dobson A. Joint modelling of longitudinal measurements and event time data. Biostatistics 2000;1:465-80.

[31] Ye W, Lin X, Taylor JMG. Semiparametric modeling of longitudinal measurements and time-to-event data - a two-stage regression calibration approach. Biometrics 2008;64:1238-46. 
[32] Xu J, Zeger SL. Joint analysis of longitudinal data comprising repeated measures and times to events. J R Stat Soc Ser C Appl Stat 2001;50:375-87.

[33] Witteman JCM, Grobbee DE, Valkenburg HA, Vanhemert AM, Stijnen T, Burger H, et al. J-shaped relation between change in diastolic blood pressure and progression of aortic atherosclerosis. Lancet 1994;343:504-7.

[34] Allison DB, Faith MS, Heo M, Kotler DP. Hypothesis concerning the U-shaped relation between body mass index and mortality. Am J Epidemiol 1997;146:339-49.

[35] Boutitie F, Gueyffier F, Pocock S, Fagard R, Boissel JP. J-shaped relationship between blood pressure and mortality in hypertensive patients: new insights from a meta-analysis of individual-patient data. Ann Intern Med 2002;136:438-48.

[36] Kulminski AM, Arbeev KG, Kulminskaya IV, Ukraintseva SV, Land K, Akushevich I, et al. Body mass index and nine-year mortality in disabled and nondisabled older US individuals. J Am Geriatr Soc 2008;56:105-10.

[37] Kuzuya M, Enoki H, Iwata M, Hasegawa J, Hirakawa Y. J-shaped relationship between resting pulse rate and all-cause mortality in community-dwelling older people with disabilities. J Am Geriatr Soc 2008;56:367-8.

[38] Mazza A, Zamboni S, Rizzato E, Pessina AC, Tikhonoff V, Schiavon L, et al. Serum uric acid shows a J-shaped trend with coronary mortality in non-insulin-dependent diabetic elderly people. The CArdiovascular STudy in the ELderly (CASTEL). Acta Diabetol 2007;44:99-105.

[39] Okumiya K, Matsubayashi K, Wada T, Fujisawa M, Osaki Y, Doi Y, et al. A U-shaped association between home systolic blood pressure and four-year mortality in community-dwelling older men. J Am Geriatr Soc 1999;47:1415-21.

[40] Protogerou AD, Safar ME, Iaria P, Safar H, Le Dudal K, Filipovsky J, et al. Diastolic blood pressure and mortality in the elderly with cardiovascular disease. Hypertension 2007;50:172-80.

[41] Troiano RP, Frongillo EA, Sobal J, Levitsky DA. The relationship between body weight and mortality: a quantitative analysis of combined information from existing studies. Int J Obesity 1996;20:63-75.

[42] Yashin AI, Ukraintseva SV, De Benedictis G, Anisimov VN, Butov AA, Arbeev K, et al. Have the oldest old adults ever been frail in the past? A hypothesis that explains modern trends in survival. J Gerontol A Biol Sci Med Sci 2001;56:B432-42.

[43] van Uffelen JGZ, Berecki-Gisolf J, Brown WJ, Dobson AJ. What is a healthy body mass index for women in their seventies? Results from the Australian longitudinal study on women's health. J Gerontol A Biol Sci Med Sci 2010;65:844-50.

[44] Woodbury MA, Manton KG. A random-walk model of human mortality and aging. Theor Popul Biol 1977;11:37-48.

[45] Yashin AI. Dynamics in survival analysis: conditional Gaussian property vs. Cameron-Martin formula. In: Krylov NV, Lipster RS, Novikov AA, editors. Statistics and control of stochastic processes. New York: Springer; 1985. p. 446-75.

[46] Yashin AI, Manton KG, Vaupel JW. Mortality and aging in a heterogeneous population: a stochastic process model with observed and unobserved variables. Theor Popul Biol 1985;27:154-75.

[47] Yashin AI, Manton KG. Effects of unobserved and partially observed covariate processes on system failure: a review of models and estimation strategies. Statist Sci 1997;12:20-34.

[48] Manton KG, Yashin AI. Mechanisms of aging and mortality: a search for new paradigms. Odense monograph on population aging, vol. 7. Odense, Denmark: Odense University Press; 2000.

[49] Yashin AI. Conditional Gaussian estimation of dynamic system response on the basis of jerky observations. Autom Remote Control 1980;41:618-26.

[50] Seeman TE, McEwen BS, Rowe JW, Singer BH. Allostatic load as a marker of cumulative biological risk: MacArthur studies of successful aging. Proc Natl Acad Sci USA 2001;98:4770-5.

[51] Troncale JA. The aging process: physiologic changes and pharmacologic implications. Postgrad Med 1996;99:111-4, 120-2.

[52] Lund J, Tedesco P, Duke K, Wang J, Kim SK, Johnson TE. Transcriptional profile of aging in C-elegans. Curr Biol 2002;12:1566-73.

[53] Hall DM, Xu L, Drake VJ, Oberley LW, Oberley TD, Moseley PL, et al. Aging reduces adaptive capacity and stress protein expression in the liver after heat stress. J Appl Physiol 2000;89:749-59.

[54] Rankin MM, Kushner JA. Adaptive beta-cell proliferation is severely restricted with advanced age. Diabetes 2009;58:1365-72.

[55] Strehler B. Time, cells, and aging. London: Academic Press; 1962.

[56] Semenchenko GV, Khazaeli AA, Curtsinger JW, Yashin AI. Stress resistance declines with age: analysis of data from a survival experiment with Drosophila melanogaster. Biogerontology 2004;5:17-30.

[57] Ukraintseva SV, Yashin AI. Individual aging and cancer risk: how are they related? Demogr Res 2003;9:163-96.

[58] Yashin AI, Arbeev KG, Akushevich I, Kulminski A, Akushevich L, Ukraintseva SV. Stochastic model for analysis of longitudinal data on aging and mortality. Math Biosci 2007;208:538-51.

[59] Yashin AI, Ukraintseva SV, Arbeev KG, Akushevich I, Arbeeva LS, Kulminski AM. Maintaining physiological state for exceptional survival: what is the normal level of blood glucose and does it change with age? Mech Ageing Dev 2009;130:611-8.

[60] Yashin AI, Arbeev KG, Akushevich I, Ukraintseva V, Kulminski A, Arbeeva LS, et al. Exceptional survivors have lower age trajectories of blood glucose: lessons from longitudinal data. Biogerontology 2010;11:257-65.

[61] Yashin AI, Arbeev KG, Ukraintseva SV, Akushevich I, Kulminski A. Patterns of aging related changes on the way to 100: An approach to studying aging, mortality, and longevity from longitudinal data. In: 2011 living to 100 monograph, Society of Actuaries Monograph M-LI11-1. Schaumburg, IL: Society of Actuaries; 2011.

[62] Arbeev KG, Ukraintseva SV, Akushevich I, Kulminski AM, Arbeeva LS, Akushevich L, et al. Age trajectories of physiological indices in relation to healthy life course. Mech Ageing Dev 2011;132:93-102.

[63] Yashin AI, Arbeev KG, Kulminski A, Akushevich I, Akushevich L, Ukraintseva SV. Health decline, aging and mortality: how are they related? Biogerontology 2007;8:291-302.

[64] Yashin AI, Arbeev KG, Kulminski A, Akushevich I, Akushevich L, Ukraintseva SV. What age trajectories of cumulative deficits and medical costs tell us about individual aging and mortality risk: findings from the NLTCS-Medicare data. Mech Ageing Dev 2008;129:191-200.

[65] Yashin AI, Manton KG, Stallard E. Dependent competing risks: a stochastic process model. J Math Biol 1986;24:119-40. 
[66] Akushevich I, Arbeev K, Ukraintseva S, Yashin A. Theory of individual health histories and dependent competing risks. In: JSM proceedings, section on risk analysis. Alexandria, VA: American Statistical Association; 2011. p. 5385-99.

[67] Yashin AI, Arbeev KG, Akushevich I, Kulminski A, Akushevich L, Ukraintseva SV. Model of hidden heterogeneity in longitudinal data. Theor Popul Biol 2008;73:1-10.

[68] Arbeev KG, Akushevich I, Kulminski AM, Arbeeva LS, Akushevich L, Ukraintseva SV, et al. Genetic model for longitudinal studies of aging, health, and longevity and its potential application to incomplete data. J Theoret Biol 2009;258:103-11.

[69] Yashin AI, Akushevich I, Arbeev KG, Kulminski A, Ukraintseva S. Joint analysis of health histories, physiological states, and survival. Math Popul Stud 2011;18:207-33.

[70] Yashin AI, Akushevich I, Arbeev KG, Kulminski A, Ukraintseva SV. New approach for analyzing longitudinal data on health, physiological state, and survival collected using different observational plans. In: JSM proceedings, section on government statistics. Alexandria, VA: American Statistical Association; 2011. p. 5336-50.

[71] Manton KG, Stallard E, Woodbury MA, Dowd JE. Time-varying covariates in models of human mortality and aging: multidimensional generalizations of the Gompertz. J Gerontol 1994;49:B169-90.

[72] McEwen BS, Wingfield JC. The concept of allostasis in biology and biomedicine. Horm Behav 2003;43:2-15.

[73] Yashin AI, Manton KG, Stallard E. Evaluating the effects of observed and unobserved diffusion processes in survival analysis of longitudinal data. Math Model 1986;7:1353-63.

[74] Samuelson PA. Rational theory of warrant pricing. Ind Manage Rev 1965;6:13-31.

[75] Black F, Scholes M. Pricing of options and corporate liabilities. J Polit Econ 1973;81:637-54.

[76] Merton RC. Theory of rational option pricing. Bell J Econ 1973;4:141-83.

[77] Dawber TR, Meadors GF, Moore FE. Epidemiological approaches to heart disease: the Framingham study. Am J Publ Health 1951;41:279-86.

[78] Dawber TR. The Framingham study: the epidemiology of atherosclerotic disease. Cambridge, MA: Harvard University Press; 1980.

[79] Economos AC. Rate of aging, rate of dying and the mechanism of mortality. Arch Gerontol Geriatr 1982;1:3-27.

[80] Yashin AI, Ukraintseva SV, Boiko SI, Arbeev KG. Individual aging and mortality rate: how are they related? Soc Biol 2002;49:206-17.

[81] Vaupel JW, Manton KG, Stallard E. Impact of heterogeneity in individual frailty on the dynamics of mortality. Demography 1979;16:439-54.

[82] Vaupel JW, Yashin AI. Heterogeneity's ruses: some surprising effects of selection on population dynamics. Amer Statist 1985;39:176-85.

[83] Rozing MP, Westendorp RGJ. Parallel lines: nothing has changed? Aging Cell 2008;7:924-7.

[84] Koopman JJE, Rozing P, Kramer A, de Jager DJ, Ansell D, De Meester JMJ, et al. Senescence rates in patients with end-stage renal disease: a critical appraisal of the Gompertz model. Aging Cell 2011;10:233-8. 